\title{
Comment on "Vitamin D status influences cytokine production and MALAT1 expression from the PBMCs of patients with coronary artery disease and healthy controls"
}

\author{
Tinghui $\mathrm{Li}^{1} \mathbb{D}$, Lianping $\mathrm{He}^{1 *}$
}

Dear Editor,

We were glad to read the interesting study published by NowrouziSohrabi et al. ${ }^{1}$ and his study team. They revealed that in vitamin D deficient individuals a decreased level of long non-coding RNA metastasis-associated lung adenocarcinoma transcript 1 (lncRNA MALAT1) was related to cluster of differentiation (CD) 36 expression and increased interleukin(IL)-22 production. Vitamin D supplementation may act as a part to reduce MALAT1/CD36/IL-22 mediated complications, such as type 2 diabetes mellitus (T2DM) and coronary artery disease (CAD), especially in vitamin $\mathrm{D}$ deficiency. Although this study is of great significance for the prevention and treatment of vascular complications, there are some issues should be further discussed.

To begin with, statistical methods should be included in the methods section. We suggest that the statistical methods used in this study should be supplemented in the method section of the summary. It will also be helpful to explain in detail the specific methods used in each data analysis, such as the categorical data were tested by Fisher exact test and presented by frequency and percentile among others. The purpose of this study is to investigate the lncRNA MALAT1 expression and its role in cytokine production from peripheral blood mononuclear cells (PBMCs) in patients with CAD and non-CAD (NCAD) participants. However, the samples collected in this survey report are non-random samples, which may lead to biases in the experimental conclusions. And the limitation of cross-sectional study is that causal inference cannot be made. Therefore, it cannot be simply concluded that vitamin D supplementation can increase the level of MALAT1 in PBMCs, nor can it simply establish a relationship between the level of IL-6 in PBMCs and CD36.

In addition, we could not find Table 1 mentioned in the conclusion of this article, please confirm whether you have missed it. Furthermore, we cannot find the Table $S 1$ mentioned in your results and the supplementary materials you mentioned. What's more, "association between clinical and biochemical characteristics with MALAT1" cannot be found either. We found that A and $\mathrm{D}$ in Figure 1 lack legends, please confirm whether the legends of these two parts are the same as the other legends in Figure 1. Last but not least, we suggest providing detailed demographic characteristics of the sample, such as occupation, gender, and home address, to analyze whether the conclusions of this experiment are dependent on occupation or another aspect.

\section{AUTHORS' CONTRIBUTIONS}

THL: Conceptualization, Data Curation, Formal analysis, Writing - original draft, Writing - review \& editing. LPH: Conceptualization, Data curation, Formal analysis, Writing original draft, Writing - review \& editing.

\section{REFERENCE}

1. Nowrouzi-Sohrabi $P$, Kalani M, Izadpanah $P$, Ahmadvand $H$, Fakhour M, Fadaei R, et al. Vitamin D status influences cytokine production and MALAT1 expression from the PBMCS of patients with coronary artery disease and healthy controls. Rev Assoc Med Bras (1992). 2020;66(12):1712-7. https://doi. org/10.1590/1806-9282.66.12.1712

'Taizhou University, School of Medicine - Taizhou (Zhejiang), China.

*Corresponding author: lianpinghe@tzc.edu.cn

Conflicts of interest: the authors declare there is no conflicts of interest. Funding: none.

Received on July 17, 2021. Accepted on July 18, 2021. 